\section{An analysis of the $\mathrm{CFH} \mathrm{Y402H}$ genotype in AMD patients and controls from the UK, and response to PDT treatment}

SV Goverdhan 1,2, S Hannan², RB Newsom², AJ Luff ${ }^{2}, \mathrm{H}$ Griffiths ${ }^{1}$ and AJ Lotery ${ }^{1,2}$

\begin{abstract}
Aim Mutation in the complement factor $\mathrm{H}$ (CFH) gene is an important risk factor for age-related macular degeneration (AMD). In this study, we identified the strength of the CFH Y402H gene variant association in a UK AMD cohort and tested the hypothesis that this variant may influence the biological response of choroidal neovascularisation (CNV) following photodynamic therapy (PDT) for CNV.

Methods A total of 557 cases with AMD and 551 normal controls were genotyped for the

increased the risk of PDT $(\mathrm{OR}=5.48$, $P=0.015)$. The degree of visual loss following PDT was significantly higher in the CFH CC genotype group $(P=0.038) ; 50 \%$ of $C C$ cases $(n=13)$ and $45 \%$ of the CT cases $(n=12)$ lost 15 or more ETDRS letters at final follow-up. Conclusion In this UK cohort of AMD patients, the CFH Y402H variant was significantly enriched in patients with predominantly classic CNV. Patients homozygous for the CFH Y402H genotype seem to have worse visual acuity after PDT. Eye (2008) 22, 849-854; doi:10.1038/sj.eye.6702830; published online 27 April 2007
\end{abstract} CFH Y402H (1277 C/T) variant using the $5^{\prime}$ nuclease TaqMan assay for allelic discrimination. The CFH gene association for AMD, for the different CNV subtypes and for patients needing PDT was estimated.

Twenty-seven PDT-treated patients were followed up for 15 months with ETDRS-derived vision, clinical examination, and fundus angiography. Individuals with different $\mathrm{CFH}$ genotypes were then analysed for any association with visual change following PDT. Results The risk association for AMD with the CFH CC genotype (odd ratio $(\mathrm{OR})=3.62$, $\left.P_{c}<0.0001\right)$ was similar to that reported in other Caucasian cohorts. The magnitude and strength of this association was stronger in AREDS stages 2-4 (ORs $=4.48,2.69$, and 5.17). ORs for the risk of predominantly classic $\mathrm{CNV}$ were significantly raised for both the $\mathrm{CC}$ $(\mathrm{OR}=17.87, P<0.0001)$ and $\mathrm{CT}(\mathrm{OR}=9.06$, $P=0.0002)$ genotypes. The number of patients carrying the high-risk $C$ allele was $70.4 \%$ in those requiring PDT as compared to $52.3 \%$ in the non-PDT group ( $O R=2.16, P=0.011)$, and presence of the CC genotype significantly
Keywords: CFH; AMD; CNV; photodynamic; acuity; association

\section{Introduction}

Complement factor $\mathrm{H}(\mathrm{CFH})$, a key inhibitor of the alternative complement pathway, has been implicated in patients with age-related macular degeneration (AMD). Many key publications have reported a highly significant $\mathrm{CFH}$ gene coding polymorphism (rs1061170, Y402H), which has been seen in nearly $50 \%$ cases of AMD. ${ }^{1-4} \mathrm{~A}$ defective factor $\mathrm{H}$ protein has been hypothesised to influence AMD-related inflammatory processes including angiogenic processes leading to choroidal neovascularisation $(\mathrm{CNV}){ }^{5}$

Photodynamic therapy (PDT) is an effective treatment for AMD-related CNV. PDT produces $\mathrm{CNV}$ regression by various cellular mechanisms, which include a variety of immunological processes. Some of these may
${ }^{1}$ Clinical Neurosciences Division, University of Southampton, Southampton General Hospital, Southampton, UK

${ }^{2}$ Southampton Eye Unit, University of Southampton, Southampton General Hospital, Southampton, UK

Correspondence: AJ Lotery, Clinical Neurosciences, Mailpoint 806,

Southampton General Hospital,

Tremona Road, Southampton SO16 6YD, UK

Tel: +44 2380794590 ;

Fax: + 442380794120

E-mail: a.j.lotery@

soton.ac.uk

Received: 2 December 2006 Accepted in revised form: 9 March 2007

Published online: 27 April 2007 
influence the complement pathway. ${ }^{6}$ PDT has been shown to produce a transient inflammatory response by increasing cytokine levels. Of these, interleukin- $1 \beta$, interleukin-2, and TNF-7 $\alpha$ all seem to be important. ${ }^{6}$ Also, antigen-presenting cells (dendritic or Langerhans) have been reported to become less active while HLA class II antigens are significantly downregulated after PDT, which may also affect immune responses. ${ }^{6}$ These inflammatory responses might stimulate wound healing or paradoxically lead to recurrent CNV.

$\mathrm{CFH}$ is present in the retina of AMD patients. Immunolocalisation studies demonstrate $\mathrm{CFH}$ colocalising within drusen, the choroid bordering drusen, and the retinal pigment epithelium (RPE), and in both the basement membrane and wall of choriocapillaries. ${ }^{4}$ Functionally, a normal CFH has been shown to limit immune complex deposition and prevent inflammatory scarring in mice models of kidney disease. ${ }^{7}$ Damage to the neurosensory retina is reported as minimal and reversible after PDT. ${ }^{6}$ However, PDT may also have undesirable consequences including RPE damage and choroidal hypoperfusion. ${ }^{6,8}$ Further, the potential for collateral damage to other retinal cell types including glial cells from multiple PDT applications cannot be discounted. Inflammatory scarring may therefore be important in PDT.

We hypothesised that mutant CFH could affect PDT outcomes by altering immune- or scarring-related processes. To answer this, we first evaluated the magnitude of AMD/CNV risk conferred by the $\mathrm{CFH}$ $\mathrm{Y} 402 \mathrm{H}$ polymorphism in our UK cohort and secondly tested whether any of the high-risk CFH genotypes associated with visual outcomes following PDT for CNV in AMD patients.

\section{Materials and methods}

\section{Patients}

The study was approved by the Southampton and Southwest Hants Local Research Ethics Committee (no. 347/02/t) and followed the Declaration of Helsinki tenets. After informed and written consent, Caucasian subjects over the age of 55 years with a diagnosis of AMD and normal Caucasian controls over the age of 55 years were recruited from ophthalmology clinics at Southampton Eye Unit, UK. Patients for the study underwent a detailed ophthalmic examination to characterize AMD phenotypes. ETDRS visual acuities were recorded. Stereoscopic fundus photographs and fluorescein angiograms were recorded with a Topcon digital retinal camera (model TRC50IX). Photographs and fluorescein angiograms were graded independently by two masked observers ( $\mathrm{SG}$ and $\mathrm{SH}$ ) into four groups of increasing disease severity as described in the AREDS study. ${ }^{9}$ Information was also obtained about relevant past medical history, smoking history, ocular history, use of medications, or vitamin or dietary supplementation. Height, weight and body mass index (BMI) measurements were recorded. A $10 \mathrm{ml}$ peripheral blood sample was collected from these patients. After $30 \mathrm{~min}$, the clotted blood sample was centrifuged for $10 \mathrm{~min}$ at $1000 \mathrm{~g}$ to separate serum; DNA was extracted and stored at $-20^{\circ} \mathrm{C}$. Twenty-seven patients who underwent PDT treatments were identified from the AMD group and followed up during a period of 15 months.

\section{SNP genotyping assay}

Genotyping was carried out using the $5^{\prime}$ nuclease assay for allelic discrimination. Primers and individual fluorogenic TaqMan probes (consisting of an oligonucleotide labelled with both a fluorescent reporter dye $-\mathrm{FAM}^{\mathrm{TM}}$ and a quencher dye $-\mathrm{VIC}^{\circledR}$ ) were designed using Primer Express software (version 2.0; sequences shown in Table 1). These were then obtained from Applied Biosystems, UK. All assays were performed using the supplied Q-PCR buffer $(2.5 \mu \mathrm{l}), \mathrm{PCR}$ probe mix $(0.125 \mu \mathrm{l})$, and $20 \mathrm{ng}$ of genomic DNA in $2.375 \mu \mathrm{l} \mathrm{dH_{2 }} \mathrm{O}$ to make a $5 \mu \mathrm{l}$ reaction volume (384-well plate). The PCR thermocycling protocol consisted of $10 \mathrm{~min}$ at $95^{\circ} \mathrm{C}$, followed by 40 cycles of $15 \mathrm{~s}$ at $92^{\circ} \mathrm{C}$, and $1 \mathrm{~min}$ at $60^{\circ} \mathrm{C}$. Each genotyping plate contained eight wells without any DNA template (water controls) and randomly selected duplicate samples $(10 \%$ of plate samples). Allelic level genotyping from fluorescence measurements was then obtained using the ABI PRISM ${ }^{\circledR}$

Table 1 Primer and probe sequences used for $5^{\prime}$ nuclease (TaqMan) genotyping

\begin{tabular}{ll}
\hline Primer/probe & Sequence \\
\hline CFH 1277 forward & $5^{\prime}$-CCATGCCTCAGAAAATGTTATTTTCCTT-3' \\
CFH 1277 reverse & $5^{\prime}$-GGCAGGCAACGTCTATAGATTTACC-3' \\
CFH 1277 C probe & VIC-TTTCTTCCATGATTTTG-MGB \\
CFH 1277 T probe & FAM-TTCTTCCATAATTTTG-MGB \\
\hline
\end{tabular}

Forward and reverse sequences are primers. VIC and FAM probe sequences have positions of single-nucleotide polymorphism marked in bold case. MGB, minor groove-binding probe. 
7900HT Sequence Detection System. SDS version 2.1 software was used to analyse real-time and end-point fluorescence data. All duplicate results were concordant.

\section{Statistical methods}

Genotype distributions for the $\mathrm{CFH}$ polymorphism were tested for conformity with Hardy-Weinberg equilibrium in both cases and controls. Alleles at each locus were said to be in Hardy-Weinberg equilibrium if the observed homozygote and heterozygote frequencies did not differ significantly $(P>0.05)$ from expected frequencies. Individual $\mathrm{CFH}$ genotypes were analysed for AMD/CNV association using Fisher's exact two-tailed tests. The association with degree of visual loss following PDT therapy was analysed by non-parametric Mann-Whitney tests. Logistic regression analysis was performed using data on age, sex, BMI, and smoking status as covariates in regression models. The level of statistical significance in the study was set at $P_{C}<0.05$. All statistical analyses were performed using the SPSS statistical software package version 14.0.

\section{Results}

Baseline demographics of the unrelated Caucasian cohort recruited from a clinic population (557 cases with AMD, 551 normal controls, and 27 cases from the AMD group who underwent PDT) are described in Table 2. CFH 1277 $\mathrm{C} / \mathrm{T}(\mathrm{Y} 402 \mathrm{H})$ genotyping data were available for all

Table 2 Basic demographic characteristics of the Caucasian study population

\begin{tabular}{lccc}
\hline & $\begin{array}{c}\text { AMD cases, } \\
\text { total } \mathrm{n}=557 \\
(\%)\end{array}$ & $\begin{array}{c}\text { Controls, total } \\
\mathrm{n}=551(\%)\end{array}$ & $\begin{array}{c}\text { PDT cases, } \\
\text { total } \mathrm{n}=27 \\
(\%)\end{array}$ \\
\hline Age & $n=557$ & $n=551$ & $n=27$ \\
Mean & 78.4 & 69.1 & 78.6 \\
Range & $55-101$ & $55-91$ & $65-93$ \\
SD & 8.65 & 9.76 & 6.13 \\
& & & \\
Sex & $n=557$ & $n=551$ & $n=27$ \\
Female & $330(59.2)$ & $308(55.9)$ & $13(48.1)$ \\
Male & $227(40.8)$ & $243(44.1)$ & $14(51.8)$ \\
BMI & $n=237$ & $n=407$ & $n=24$ \\
Mean & 26.5 & 27.3 & 25.4 \\
SD & 4.76 & 4.70 & 4.98 \\
Smoking status & $n=460$ & $n=432$ & $n=27$ \\
Current & $140(30.4)$ & $81(18.7)$ & $8(29.9)$ \\
smokers & & & \\
Past smokers & $145(31.5)$ & $213(49.3)$ & $12(44.4)$ \\
Non-smokers & $175(38.0)$ & $138(31.9)$ & $7(25.7)$ \\
\hline
\end{tabular}

individuals. Genotype frequency distributions were as shown in Table 3. The homozygous CC genotype was more often present in AMD cases ( 0.28 vs 0.13 ,

$P<0.0001)$ giving an odds ratio (OR) for disease of 3.62 . The association with AMD was also significant in the larger heterozygous CT group but with a lesser risk of AMD than in the CC group (OR=1.61, $P=0.0008)$. Overall, the OR for AMD was 1.90 for the high-risk $C$ allele $(<0.0001)$. Adjusting for age during the logistic regression analysis did not alter the OR for disease significantly.

Further categorisation was possible in 416 subjects in the AMD group, who were divided into four groups of increasing AMD severity using AREDS criteria (Table 4). The homozygous CC genotype was found to associate with all the four AREDS groups with the strongest association seen in the AREDS-4 group (OR $=5.17$, $P<0.0001$ ). Although the OR in AREDS-1 was similar to that in AREDS-3 (OR of 2.64 vs 2.69), the strength of this association in AREDS- 1 was much weaker $(P=0.03$ vs $P=0.0008$ ). The heterozygous CT genotype was found to associate with AREDS-4 group only $(\mathrm{OR}=1.78$, $P=0.008$ ). The $\mathrm{C}$ allele showed a similar risk trend for association in all the four AREDS groups with ORs ranging from 1.61 to 2.33 .

The risk of various subtypes of $\mathrm{CNV}$ was then analysed in comparison to controls (Table 5). CFH genotypes were found to associate with the predominantly classic CNV group only (OR for CC genotype $=17.87, P<0.0001, \mathrm{OR}$ for $\mathrm{CT}$ genotype $=9.06$, $P=0.0002$, combined $\mathrm{OR}=10.87, P<0.0001)$. OR for the presence of the high-risk $C$ allele was $2.97(P<0.0001)$ in this CNV subtype. Allele associations were also seen in the occult $\mathrm{CNV}(\mathrm{OR}=3.09, P<0.0001)$ and minimally classic $\mathrm{CNV}(\mathrm{OR}=2.52, P=0.001)$ subtypes; this was however not significant when genotypes were analysed in these groups.

Table 3 CFH Y402H genotype association in AMD cases and controls

\begin{tabular}{|c|c|c|c|}
\hline & $\begin{array}{c}A M D \\
\text { cases, } \\
\mathrm{n}=557 \\
(\%)\end{array}$ & $\begin{array}{c}\text { Controls, } \\
\mathrm{n}=551 \\
(\%)\end{array}$ & $\begin{array}{l}\text { Genotype association, } \\
\text { OR, } 95 \% \text { CI }\left(\mathrm{P}_{\left.C^{-}-\text {value }\right)^{\mathrm{a}}}\right.\end{array}$ \\
\hline \multicolumn{4}{|c|}{ CFH genotypes } \\
\hline $\mathrm{CC}$ & $167(0.28)$ & $75(0.13)$ & $3.62,2.56-5.14(<0.0001)$ \\
\hline CT & $258(0.44)$ & $261(0.44)$ & $1.61,1.22-2.12(0.0008)$ \\
\hline $\mathrm{TT}$ & $132(0.23)$ & $215(0.36)$ & - \\
\hline \multicolumn{4}{|c|}{ CFH allele } \\
\hline $\mathrm{C}$ & 592 & $411(0.37)$ & $1.90,1.60-2.25(<0.0001)$ \\
\hline $\mathrm{T}$ & 522 & $691(0.63)$ & - \\
\hline
\end{tabular}

${ }^{\mathrm{a}} P_{\mathrm{C}}=P$-values for genotypes (compared to TT) after adjusting for age, sex, BMI, current smokers, and past smokers. 
Table 4 CFH Y402H genotype association in the AREDS subtypes compared to controls

\begin{tabular}{|c|c|c|c|c|c|}
\hline & $\begin{array}{l}\text { AREDS-1, } \mathrm{n}=51(\%) \\
\quad(O R, \mathrm{P} \text {-value, } C I)^{\mathrm{a}}\end{array}$ & $\begin{array}{l}\text { AREDS-2, } \mathrm{n}=73(\%) \\
\quad(\text { OR, P-value, } C I)^{\mathrm{a}}\end{array}$ & $\begin{array}{l}\text { AREDS-3, } \mathrm{n}=113(\%) \\
\quad(\text { OR, } \mathrm{P} \text {-value, } C I)^{\mathrm{a}}\end{array}$ & $\begin{array}{l}\text { AREDS-4, } \mathrm{n}=179(\%) \\
(\text { OR, P-value, } C I)^{\mathrm{a}}\end{array}$ & $\begin{array}{c}\text { Controls, } \\
\mathrm{n}=551(\%)\end{array}$ \\
\hline \multicolumn{6}{|c|}{ CFH Genotype } \\
\hline $\mathrm{CC}$ & $\begin{array}{c}12(23.5) \\
(2.64, P=0.03,1.16-6.05)^{\mathrm{a}}\end{array}$ & $\begin{array}{c}25(34.2) \\
(4.48, P<0.0001,2.27-8.84)^{\mathrm{a}}\end{array}$ & $\begin{array}{c}31(27.4) \\
(2.69, P=0.0008,1.54-4.69)^{\mathrm{a}}\end{array}$ & $\begin{array}{c}65(36.3) \\
(5.17, P<0.0001,3.19-8.40)^{\mathrm{a}}\end{array}$ & $75(0.13)$ \\
\hline $\mathrm{CT}$ & $26(51.0)$ & $32(43.8)$ & $49(43.4)$ & $\begin{array}{c}78(43.6) \\
(1.78, P=0.008,2.59-16.54)^{\mathrm{a}}\end{array}$ & $261(0.44)$ \\
\hline $\mathrm{TT}$ & $13(25.5)$ & $16(22.0)$ & $33(29.2)$ & $36(20.1)$ & $215(0.36)$ \\
\hline \multicolumn{6}{|c|}{ CFH allele } \\
\hline C & $\begin{array}{c}50(0.49) \\
(1.61, P=0.02,1.07-2.42)^{\mathrm{a}}\end{array}$ & $\begin{array}{c}82(0.56) \\
(2.15, P<0.0001,1.51-3.05)^{\mathrm{a}}\end{array}$ & $\begin{array}{c}111(0.49) \\
(1.62, P=0.001,1.21-2.16)^{\mathrm{a}}\end{array}$ & $\begin{array}{c}208(0.58) \\
(2.33, P<0.0001,1.82-2.97)^{\mathrm{a}}\end{array}$ & $411(0.37)$ \\
\hline $\mathrm{T}$ & $52(0.51)$ & $64(0.44)$ & $115(0.51)$ & $150(0.42)$ & $691(0.63)$ \\
\hline
\end{tabular}

${ }^{a} P$-values by Fisher's exact test (two-sided values), only significant values are shown.

Table 5 Risk for CNV subtypes associated with CFH Y402H genotypes and alleles in comparison to controls

\begin{tabular}{|c|c|c|c|c|c|c|}
\hline & $\begin{array}{l}\text { Occult } \mathrm{CNV}, \mathrm{n}=49(\%) \\
(\text { OR, P-value, } 95 \% C I)^{\mathrm{a}}\end{array}$ & $\begin{array}{l}\text { Serous PED, } \\
\mathrm{n}=12(\%)\end{array}$ & $\begin{array}{l}\text { Minimally classic, } \\
\mathrm{n}=25(\%)\end{array}$ & $\begin{array}{c}\text { Predominantly classic, } \\
\mathrm{n}=36(\%) \\
(\text { OR, P-value, } 95 \% C I)^{\mathrm{b}}\end{array}$ & $\begin{array}{c}\text { Classic, } \mathrm{n}=17 \\
(\%)\end{array}$ & $\begin{array}{c}\text { Controls, } \mathrm{n}=551 \\
(\%)\end{array}$ \\
\hline \multicolumn{7}{|c|}{ CFH genotype } \\
\hline $\mathrm{CC}$ & $21(42.9)$ & $4(33.3)$ & $10(40.0)$ & $\begin{array}{c}12(33.3) \\
(17.87, P<0.0001,3.76-78.66)^{\mathrm{a}}\end{array}$ & $2(11.8)$ & $75(0.13)$ \\
\hline $\mathrm{CT}$ & 15 (30.6) & $5(41.7)$ & $10(40.0)$ & $\begin{array}{c}22(61.2) \\
(9.06, P=0.0002,2.10-38.98)^{\mathrm{a}}\end{array}$ & $9(52.9)$ & $261(0.44)$ \\
\hline TT & $13(26.5)$ & $3(25.0)$ & $5(20.0)$ & $2(5.5)$ & $6(35.3)$ & $215(0.36)$ \\
\hline \multicolumn{7}{|c|}{ CFH allele } \\
\hline $\mathrm{C}$ & $\begin{array}{c}57(64.7) \\
(3.09, P<0.0001,1.96-4.86)^{\mathrm{a}}\end{array}$ & $13(54.2)$ & $\begin{array}{c}30(60.0) \\
(2.52, P=0.001 \\
1.41-4.50)^{\mathrm{a}}\end{array}$ & $\begin{array}{c}46(63.9) \\
(2.97, P<0.0001,1.81-4.88)^{\mathrm{a}}\end{array}$ & $13(38.2)$ & 411 (37.0) \\
\hline $\mathrm{T}$ & $31(35.3)$ & $11(45.8)$ & $20(40.0)$ & $26(36.1)$ & $21(62.8)$ & $691(63.0)$ \\
\hline
\end{tabular}

${ }^{a} P$-values by Fisher's exact test (two-sided values comparing CC/CT $v s$ TT genotypes and $\mathrm{C} v s \mathrm{~T}$ alleles).

${ }^{\mathrm{b}} \mathrm{C}$ ombined OR for CC $+\mathrm{CT}$ in predominantly classic $=10.87, P<0.0001,95 \% \mathrm{CI}=2.58-45.76$. Only significant values are shown. 
The risk of developing classic or predominantly CNV within the wet AMD group was then compared to the minimally classic and occult CNV (including PED) group. Analysis was performed for association with $\mathrm{CFH}$ genotypes (CC/CT) and alleles; no significant difference in the frequency of $\mathrm{CFH}$ genotypes or alleles was found between the two groups (data not shown).

Overall, 76\% (425/557) of AMD patients had the CC or CT genotype (Table 6 ). Of these, $4.8 \%$ patients $(n=27 / 557)$ had undergone PDT treatments. In the PDT group, the number of patients carrying the high-risk $\mathrm{C}$ allele was $70.4 \%$ as compared to $52.3 \%$ in the non-PDT group $(\mathrm{OR}=2.16, P=0.011)$. This difference in the genotype distributions in the PDT and non-PDT groups was most significant for the CC genotype (49 vs $29 \%$, $\mathrm{OR}=5.48, P=0.015)$.

In the PDT group, 27 patients who had predominantly classic or $100 \%$ classic CNV (Snellen VA $\geqslant 6 / 60$ ) were followed after PDT for a period ranging from 3 to 22 months (median $=22$ months). Each patient on average had received at least two PDT treatments (median $=2$, range $=1-5$ treatments). Other demographics of this PDT cohort are described in Table 2. In this group, the CC and CT were the predominant genotypes observed in 48 and $44 \%$ cases, respectively.

Visual acuity data after PDT for each genotype are presented in Table 7. Overall, $48 \%$ of CC cases $(n=13)$ and $44 \%$ of the CT cases $(n=12)$ had lost 15 or more ETDRS letters. The numbers were too small in the TT group for analysis $(n=2)$. The degree of ETDRS acuity loss was statistically significant in the CC group (median loss of 12 letters, $P=0.038$ ). The loss of ETDRS letters in the CT group was not significant (median loss of 3.5 letters, $P=0.087$ ). Although acuity loss was greatest in the TT group (median loss of 70 letters), the sample size was small and $P$-values were not obtainable.

\section{Discussion}

$\mathrm{CFH}$ is an essential inhibitor of the alternative complement pathway preventing uncontrolled complement activation. ${ }^{10}$ Recent data also suggest that the CFH Y402H polymorphism is a risk factor for several AMD phenotypes including CNV, geographic atrophy, and soft drusen. ${ }^{11,12}$ This study has sought to identify the risk of $\mathrm{AMD}$ and particularly $\mathrm{CNV}$ in a UK cohort conferred by the presence of this CFH polymorphism additionally exploring any effect of these $\mathrm{CFH}$ genotypes on PDT outcomes.

In this UK study, the strength of the AMD association with the high-risk CC and the heterozygous CT genotypes was similar to that reported in other Caucasian cohorts. The CC genotype showed a five-fold risk in the AREDS-4 group compared to controls. Within
Table $6 \mathrm{CFH}$ Y402H risk genotypes (CC and CT) in PDT and non-PDT groups

\begin{tabular}{|c|c|c|c|}
\hline & $\begin{array}{c}\text { PDT group, } \\
\mathrm{n}=27(\%)\end{array}$ & $\begin{array}{c}\text { Non-PDT } \\
\text { group } \\
\mathrm{n}=530(\%)\end{array}$ & $\begin{array}{l}\text { Genotype association } \\
(\text { OR, P-value, } 95 \% C I)^{\mathrm{a}}\end{array}$ \\
\hline \multicolumn{4}{|c|}{ CFH genotype } \\
\hline $\mathrm{CC}^{\circ}$ & $13(49.0)$ & $154(29.0)$ & $5.48,0.015,1.21-24.77^{\mathrm{b}}$ \\
\hline $\mathrm{CT}$ & $12(44.0)$ & $246(46.4)$ & $3.17,0.15,0.69-14.38$ \\
\hline TT & $2(7.0)$ & $130(24.6)$ & - \\
\hline \multicolumn{4}{|c|}{ CFH allele } \\
\hline $\mathrm{C}$ & $38(70.4)$ & $554(52.3)$ & $2.16,0.011,1.19-3.93^{b}$ \\
\hline $\mathrm{T}$ & $16(29.6)$ & $506(47.7)$ & - \\
\hline
\end{tabular}

Table 7 CFH Y402H risk genotypes (CC and CT) and visual acuity following PDT

\begin{tabular}{lccc}
\hline & $\begin{array}{c}\text { PDT cases } \\
\mathrm{n}=27\end{array}$ & $\begin{array}{c}\text { ETDRS acuity } \\
\text { loss } \\
\text { (median, range) }\end{array}$ & $\begin{array}{c}\text { Genotype vs } \\
\text { ETDRS acuity } \\
\text { loss (P-value) }\end{array}$ \\
\hline CFH genotype & $13(0.48)$ & $-12.0(+23.0$ to -70.0$)$ & $P=0.038^{\mathrm{a}}$ \\
CC & $12(0.44)$ & $-3.5(+20.0$ to -80.0$)$ & $P=0.087$ \\
CT & $2(0.08)$ & $-70.0(-65.0$ to -75.0$)$ & - \\
TT &
\end{tabular}

aSignificant $P$-values (CC and CT genotypes compared to TT genotype using non-parametric Mann-Whitney test).

the AMD group, the association of predominantly classic $\mathrm{CNV}$ with the CFH C allele was highly significant. The magnitude of this risk was five times greater in patients with CC or CT genotypes. When compared to the control group, the risk of predominantly classic CNV further increased by 17-fold in the CC group and 9-fold in the CT group. Recently, Haines et $a l^{1}$ reported similar raised ORs of 3.4 and 5.6 for the CT and CC genotypes when their analysis was restricted to CNV. Zareparsi et $a l^{13}$ noted a similar raised frequency of CC genotypes in $\mathrm{CNV}$. In a recent report by Sepp et al, ${ }^{11} 81.8 \%$ of AMD patients with $\mathrm{CNV}$ were found to exhibit the high-risk $\mathrm{CFH}$ genotypes, the ORs for CNV being 5.1 for CC and 2.7 for CT genotypes. The reported OR for CNV risk was 4.34 in a recent report from an Australian AMD cohort. ${ }^{14}$ Our risk figures for $\mathrm{CNV}$ seem to be similar to the above reported rates. However, the correlation of CFH genotypes with predominantly classic CNV was higher than any of these reported associations. We are not aware of any previous analysis of predominantly classic or other CNV subtypes with $\mathrm{CFH}$ genotype. The higher correlation for this $\mathrm{CNV}$ type may therefore reflect a tendency for the CFH mutant protein to result in this subtype of $\mathrm{CNV}$. 
A significantly increased frequency of CC and CT genotypes $(93 \%)$ was seen in the PDT group with significantly higher proportion of patients carrying the high-risk $\mathrm{C}$ allele (70.4\%) in those requiring PDT as compared to $52.3 \%$ in the non-PDT group $(\mathrm{OR}=2.16$, $P=0.011)$ and $(\mathrm{OR}=5.48, P=0.015)$. The presence of the $\mathrm{CFH}$ CC genotype seemed to increase the risk of PDT significantly. In this study, the degree of visual loss following PDT was also found to be significantly higher in this genotype group, whereas visual acuity was unrelated to the presence of heterozygous CT and homozygous TT genotypes. Calculations revealed that the PDT sample size $(n=27)$ had $80 \%$ power to detect a minimum difference of 20 ETDRS letters (alpha error level $=5 \%$ ) and $90 \%$ power to detect a 24-letter difference. The power to detect any smaller differences in acuity would have been limited.

Although sample sizes are adequate, the power to detect the association of $\mathrm{CFH} \mathrm{Y} 402 \mathrm{H}$ genotype with predominantly classic CNV may be limited given the close prevalence of $\mathrm{CFH}$ in the various membrane types, classic, minimally classic, or occult. In addition, the type of neovascularisation may change over time in an individual and it is possible that some individuals in the AMD group would have developed some form of occult CNV making them ineligible for PDT therapy. However if so, the associations described for the predominantly classic form of CNV would be an underestimate. $\mathrm{CFH}$ risk genotype associations with predominantly classic $\mathrm{CNV}$ may therefore be stronger than we describe.

In conclusion, there was a highly significant trend for patients with the risk $\mathrm{Y} 402 \mathrm{H}$ genotypes to develop predominantly classic CNV in AMD. The CFH Y402H gene variant was found to associate with PDT treatments for CNV and also seemed to affect visual outcomes following PDT. It may be that once CNV begins, downstream events such as VEGF expression and scarring predict final visual outcome in addition to $\mathrm{CFH}$ genotype. Visual acuity responses to PDT therefore seem to be related to $\mathrm{CFH} \mathrm{Y} 402 \mathrm{H}$ genotype. This may be because of CFH-related damage to the RPE allowing CNV to develop more easily above the RPE. This is however an exploratory hypothesis testing study. Further studies are needed to confirm the association reported here of a predominantly classic CNV subtype in patients with $\mathrm{Y} 402 \mathrm{H} \mathrm{CFH}$ genotypes. Such genotype-phenotype correlations may ultimately assist in clinical treatment algorithms.

\section{Acknowledgements}

This work was supported by Wellcome Trust, Lord Sandberg, British Council Prevention Blindness. We thank the Wellcome Trust Clinical Research Facility and our patients for contributing to this research.

\section{References}

1 Haines JL, Hauser MA, Schmidt S, Scott WK, Olson LM, Gallins $\mathrm{P}$ et al. Complement factor $\mathrm{H}$ variant increases the risk of age-related macular degeneration. Science 2005; 308: 419-421.

2 Klein RJ, Zeiss C, Chew EY, Tsai JY, Sackler RS, Haynes $\mathrm{C}$ et al. Complement factor $\mathrm{H}$ polymorphism in age-related macular degeneration. Science 2005; 308 : 385-389.

3 Edwards AO, Ritter IR, Abel KJ, Manning A, Panhuysen C, Farrer LA. Complement factor H polymorphism and age-related macular degeneration. Science 2005; 15: 421-424.

4 Hageman GS, Anderson DH, Johnson LV, Hancox LS, Taiber AJ, Hardisty LI et al. A common haplotype in the complement regulatory gene factor $\mathrm{H}(\mathrm{HF} 1 / \mathrm{CFH})$ predisposes individuals to age-related macular degeneration. Proc Natl Acad Sci USA 2005; 102: 7227-7232.

5 Donoso LA, Kim D, Frost A, Callahan A, Hageman G. The role of inflammation in the pathogenesis of age-related macular degeneration. Surv Ophthalmol 2006; 51: 137-152.

6 Schmidt-Erfurth U, Hasan T. Mechanisms of action of photodynamic therapy with verteporfin for the treatment of age-related macular degeneration. Surv Ophthalmol 2000; 45: 195-214.

7 Alexander JJ, Pickering MC, Haas M, Osawe I, Quigg RJ. Complement factor $\mathrm{H}$ limits immune complex deposition and prevents inflammation and scarring in glomeruli of mice with chronic serum sickness. J Am Soc Nephrol 2005; 16: $52-57$.

8 Wachtlin J, Behme T, Heimann H, Kellner U, Foerster MH. Concentric retinal pigment epithelium atrophy after a single photodynamic therapy. Graefes Arch Clin Exp Ophthalmol 2003; 241: 518-521.

9 Age-Related Eye Disease Study Research Group. Risk factors associated with age-related macular degeneration. A case-control study in the age-related eye disease study: age-related eye disease study report number 3. Ophthalmol 2000; 107: 2224-2232.

10 Rodriguez de CS, Esparza-Gordillo J, Goicoechea de JE, Lopez-Trascasa M, Sanchez-Corral P. The human complement factor $\mathrm{H}$ : functional roles, genetic variations and disease associations. Mol Immunol 2004; 41: 355-367.

11 Sepp T, Khan JC, Thurlby DA, Shahid H, Clayton DG, Moore AT et al. Complement factor $\mathrm{H}$ variant $\mathrm{Y} 402 \mathrm{H}$ is a major risk determinant for geographic atrophy and choroidal neovascularization in smokers and nonsmokers. Invest Ophthalmol Vis Sci 2006; 47: 536-540.

12 Magnusson KP, Duan S, Sigurdsson H, Petursson H, Yang Z, Zhao Y et al. CFH Y402H confers similar risk of soft drusen and both forms of advanced AMD. PLoS Med 2006; 3: e5.

13 Zareparsi S, Branham KE, Li M, Shah S, Klein RJ, Ott J et al. Strong association of the $\mathrm{Y} 402 \mathrm{H}$ variant in complement factor $\mathrm{H}$ at $1 \mathrm{q} 32$ with susceptibility to age-related macular degeneration. Am J Hum Genet 2005; 77: 149-153.

14 Baird PN, Islam FM, Richardson AJ, Cain M, Hunt N, Guymer R. Analysis of the Y402H variant of the complement factor $\mathrm{H}$ gene in age-related macular degeneration. Invest Ophthalmol Vis Sci 2006; 47: 4194-4198. 